\title{
Communicating Mobile Robots a Novel Learning Approach
}

\author{
S.S. Shriramwar, \\ Priyadarshini College of Engg., Nagpur
}

\author{
N.K. Choudhari \\ Smt. B.Chaturvedi College of Engg. Nagpur
}

\begin{abstract}
We introduce and demonstrate here a learning and decisionmaking problem associated with a complex task that integrates human and robotic learning dynamics with feedback. This paper aims to provide a contribution to the theme of a colony of robots in the subject area of inter-agent communication and learning. The communication technique not only allows the COMBOTS i.e. communicating robots, to transmit, at regular intervals, their physiological state as well as their psychological state to the outside world in general and other robots in particular, but also makes them capable to interact with a tutor robot that makes them learn the tasks as desired. The paper demonstrates techniques which allow realistic explorations concerning the evolution of robot intelligence to respond to real world entities.
\end{abstract}

\section{Keywords}

COMBOT, Intelligence building, Robot learning ,Master, Tutor.

\section{INTRODUCTION}

Even if AI (Artificial Intelligence) was for long the champion of declarative knowledge, of learning defined by itself, irrespective of its use, it has since realized, in connection with distributed AI, that we cannot understand knowledge that has been experienced, that is, meaning, without referring to the behavior of an agent situated in an environment and in relation to other agents.[1]. Based on this, our research begins at the basic level of establishing robot communication in a multi-robot system and takes steps towards building learning robots, thus evolving into a colony of robots where there is a master or tutor robot that is capable of teaching and a school of robots that are capable of learning various tasks to be performed on large scale. This paper explores the prospects for using Artificial Life techniques to evolve programs to control physically embodied mobile robots, so that we no longer have to do it all by hand.[7]

We demonstrate here communicating robots, referred to here as COMBOTS, which form a colony of robots established to perform a particular task. This paper presents an advanced implementation of a virtual environment to develop and test communication protocols for multi-agent systems. Employing the real environment as a testing tool increases the efficiency of the evaluation process. The visual assessment outputs using a full-scale environment also has been advantageous in directing numerical analysis.[10]

In order for an agent to understand the outside world and to interact with it, an agent has knowledge representational system (KRS) is required. This knowledge can be described as the preconditions of actions. [10]. In case of humans this knowledge comes through learning. The research conducted here attempts to incorporate this knowledge in the form of intelligence in a communicating robot, through another robot, thus, creating a teaching environment through robots. The approach grew out of dissatisfactions with traditional robotics and Artificial Intelligence, which seemed unable to deliver real-time performance in a dynamic world. The key idea of the new approach is to advance both robotics and AI by considering the problems of building an formats for your particular conference. autonomous agent that physically is an autonomous mobile robot, and that carries out some useful tasks in an environment which has not been specially structured or engineered for it.[1].

While robots built on these principles have demonstrated learning calibration information, behavior coordination, and representations of the world, progress in learning new behaviors has proven more difficult [1].

Work in Artificial Life has developed techniques for evolving programs for controlling situated, but unembodied (i.e., simulated), robots. At some level one of the goals of Artificial Life is to move out of the digital medium into that of embodied systems.

\section{THE COMBOT}

The entire multi-agent system functions as described below. The global receiver is used to enable human-COMBOT interaction. The global receiver when receives a signal, sends it to the decoder which would translate it into a nibble of binary data and this is transmitted through the RF module.

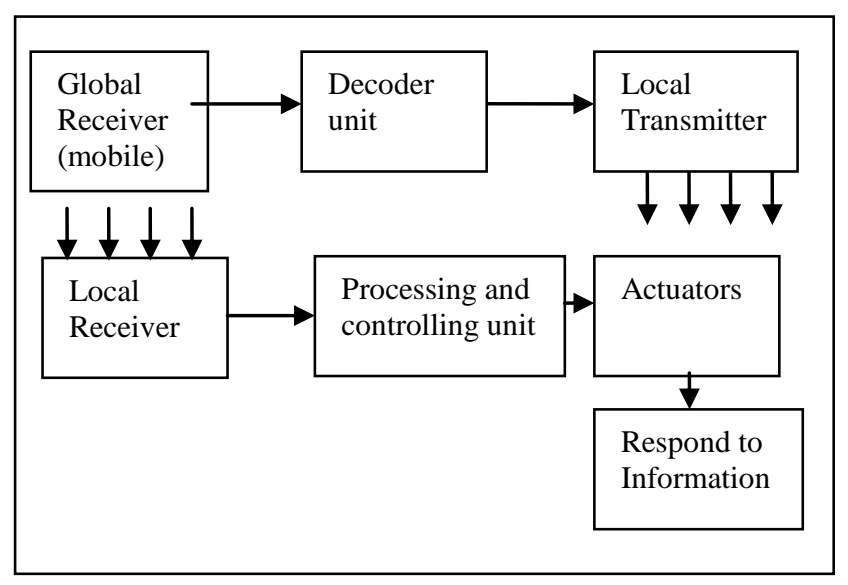

Fig 1: block diagram of the system

This transmitted data is received on the other end by the COMBOTS, both master and slaves, and is sent to the controller module where it is processed and sent to the actuation unit to enable the robot to respond accordingly.

\subsection{Hardware design}

A homogenous colony of 150 robots was designed and built to be used as physical platform in order to test a number of concepts developed in the area of multi robot systems. Specifically, the colony was built and used for testing the 
functionality and robustness of specific cognitive architecture using physical robots, validation of the results on robot colonies obtained from simulation, hardware realization of concepts in robot sensing, action, computation, and communication; and construction of a platform for further investigation of social behavior of robots.

To ensure that the removal and replacement of all components is possible as well as simple and robust for further research to proceed, the entire robot structure was made reconfigurable and circuit was implemented on breadboard. This enabled us to readily troubleshoot 150 robotic circuits and designs.

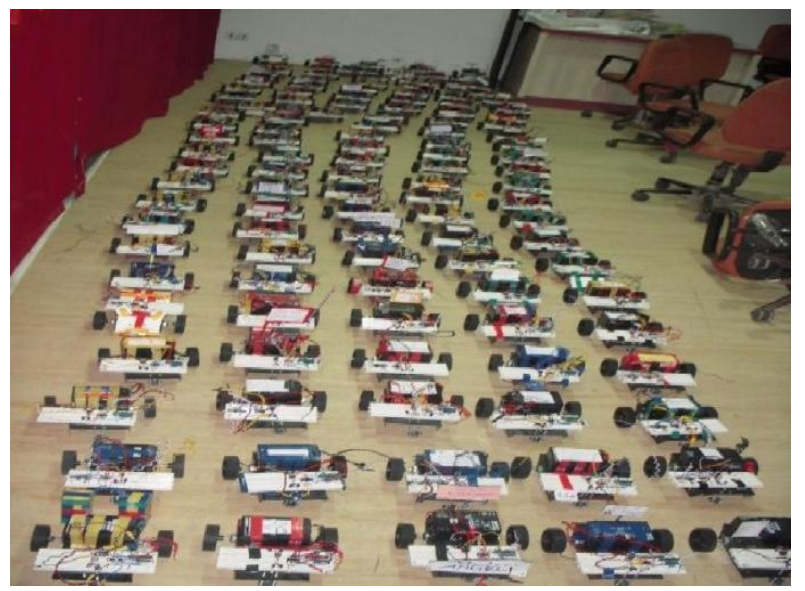

Fig 2: A colony of 150 robots

The communicating robots designed here have an RF transmitter and an RF receiver which enables it to take input and give an output wirelessly. It also consists of a controller and processing unit that provides decision-making as well as the learning capability in the robot. The actuator unit helps the COMBOT to perform the task as desired.

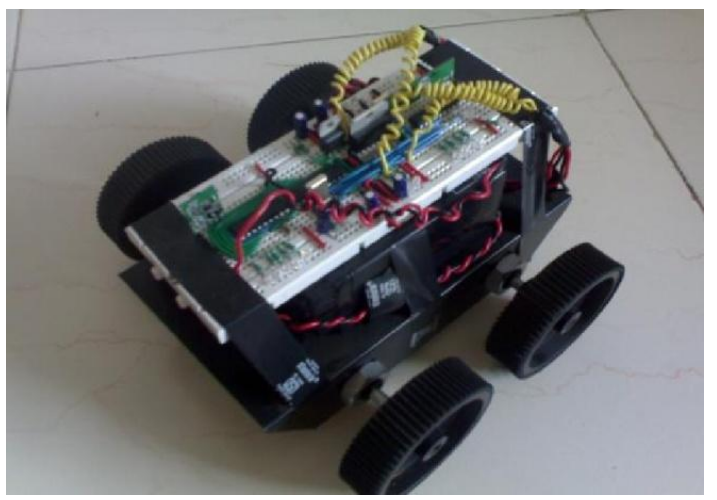

Fig. 3: Snapshot of a COMBOT

Each robot member of the colony is an electromechanical, battery-powered mobile device, controlled by an on-board micro-processor, and provided with an RF receiver and transmitter circuitry.

Each robot member of the colony is an electromechanical, battery-powered mobile device, controlled by an on-board micro-processor, and provided with an RF receiver and transmitter circuitry.

\subsection{Intelligence Building}

The multi- agent system of 150 robots was basically designed to study the single robot performance, multiple robot coperformance (implicit cooperation and implicit learning); and multiple robot communication and explicit cooperation.[2]
The purpose of the study of single robot performance is to enable the robot to learn the desired task and the functionality and robustness of the individual robots embedded within their world. The learning of the task takes place through the human interface via global receiver and decoder module. The robot is tested to survive, function, and perform tasks in the world. Apart from performing, it is also capable of learning and then teaching that particular task to the other robots, hence reducing our efforts to individually deal with all 150 robots. This performs the study of multiple robot co-performances and multiple robot communication of the multi agent system.

The following steps are performed to conduct the study:

1. We use a mobile to study the single robot performance by teaching it the sequence of steps to be taken to perform the task.

2. Once it memorizes the task, it is asked to perform it for confirming the correct transmission of data.

3. It then becomes capable of performing the task of the tutor and teaches the other robots to perform the same stepwise.

The above steps are performed with the help of mobile. [4] Each task is performed with the help of a key $\mathrm{K}$. The following algorithm describes the operation using a mobile phone.

STEP 1: transmit the code for activating a COMBOT.

STEP 2: transmit the code key $\mathrm{K}={ }^{\prime} 1{ }^{\prime}+{ }^{\prime} *$ ' to teach the tutor COMBOT, the sequence of tasks that need to be performed.

STEP 3: transmit the code key $\mathrm{K}=$ '\#' to symbolize finish teaching.

STEP 4: transmit the code key $\mathrm{K}=$ ' 2 ' + ' $*$ ' to repeat the sequence by the tutor COMBOT for verification.

STEP 5: transmit the code key $\mathrm{K}=$ ' 3 ' + '*' to enable the tutor to teach the student COMBOTS.

The key codes can be changed as desired by the programmer, they are not fixed.

\subsection{Robot Learning}

Learning can be viewed as the process of gathering information from the environment and encoding it to improve the efficiency of a system in achieving a certain goal. A computer program is said to learn from experience $\mathrm{E}$ with respect to some class of tasks $\mathrm{T}$ and performance measure $\mathrm{P}$, if its performance as tasks in $\mathrm{T}$, as measured by

$\mathrm{P}$, improves with experience E. [5] The idea behind learning is that percepts should be used not only for acting, but also for improving the agent's ability to act in the future.

In our research, the genetic programming is used to describe the artificial life through real robots. The research implements the basic symbolic learning methods and explores its application in the evolution of dynamic decision-making capabilities. It also implements the connectionist learning means and at a later stage, discusses a machine learning approach with the capabilities of both the modes along with dynamic learning.

\section{STUDENT COMBOTS}

In this section the research on COMBOT learning has focused on the learning capability based in prepositional or first-order logic. In this learning approach, an inductive and deductive reasoning was used to solve learning problems. This was achieved through manipulation of the symbolic structures that represent knowledge. This type of learning paradigm, usually referred as symbolic learning, was mainly based on mathematics, logic and some simple concepts related to psychology. 
What follows is that a systematic program is hard- coded into the decision making unit and the agent responds to a symbol according to the set of codes already provided. The following algorithm describes the code used in the first case when the COMBOT, performing as the tutor, interacts with human to learn the task it is desired to teach the rest of the COMBOTS.

STEP 1: The various tasks within the capability of the robot are programmed initially in the tutor COMBOT.

For instance, in our COMBOT, it has the following experimental capabilities:

1. Move forward with various speeds

2. Move backward with various speeds

3. Turning left and right with various speeds

4. Make a dancing pair with another COMBOT.

5. Make another COMBOT follow a virtual track.

STEP 2: The symbol for the corresponding task to be performed is transmitted.

STEP 3: The tutor COMBOT realizes the symbol and memorizes it to perform the same. STEP 4: Finally it generates a sequence of symbols until it recognizes a finish code and memorizes this sequence as the task to be performed.

In this case, the "intelligence" of these systems is hard-coded into the program, with "actual" learning taking place. This approach is successful for some small problems but difficulties start to arise when these programs are applied to larger problems. Some of the main difficulties are the exponential growth of the search space in some AI problems and the massive amount of information that had to be stored to deal with any reasonably complex problem. Still this approach serves fine for small scale learning environment.

The sequence stored in this way in the memory of the tutor COMBOT can be verified by making it to perform the same sequence with the help of key $\mathrm{K}=$ '2'+ '*'(on mobile).

After verifying the sequence, it is required for the tutor COMBOT to teach this, stepwise, to the other student COMBOTS. This is realized using the connectionist learning method in which learning is carried out by small computational units, connected by weighted links. These sets of connected units can learn to mimic, and hopefully to generalize, the behavior of a system based on its inputs and outputs (this process is usually referred as supervised learning), or to classify data according to its statistical properties. Learning is accomplished, in most of these systems, by adapting the weights of the mutual links, or the internal parameters of the units, so that an appropriate error function is minimized.

The following algorithm describes the code which enables the learning procedure by this method, in which there is an inter agent interaction to stimulate learning in the student COMBOT.

STEP 1: The tutor COMBOT performs the first sequence and transmits the command to make it do the same.

It does this by breaking down the task sequence into small weighted modules and transmitting these modules one at a time.

STEP 2: The student COMBOT receives these individual modules and forms a link between these, hence generating a class of task

For instance if the task of moving forward, in our COMBOT, has to be performed, the tutor COMBOT first moves forward itself and transmits the code which is recognized for moving forward.

STEP 3: The student COMBOT mimics the behavior of the tutor and stores the code that has been transmitted.

STEP 4: It forms the link between the two and stores it.

STEP 5: The tutor retransmits the same three times to avoid any misinterpretations.

STEP 6: Having finished the tutor transmits the finish code, on receiving which, the student learns and verifies the task by itself.

This approach although implements the actual concept of learning, it requires an extra hardware components on the student COMBOTS, to recognize the motion of the tutor. In our case the IR sensors served good to detect the motion of the wheels.

A disadvantage of these systems is that they operate as black boxes and it is difficult to generate explanations for the conclusions reached. This type of learning is rooted in signal processing, statistics as well as in some simplified concepts of biology. Despite its disadvantages this approach has produced several good results. Applications for speech recognition, scheduling and machinery control that embody these technologies are still in use today. [8]

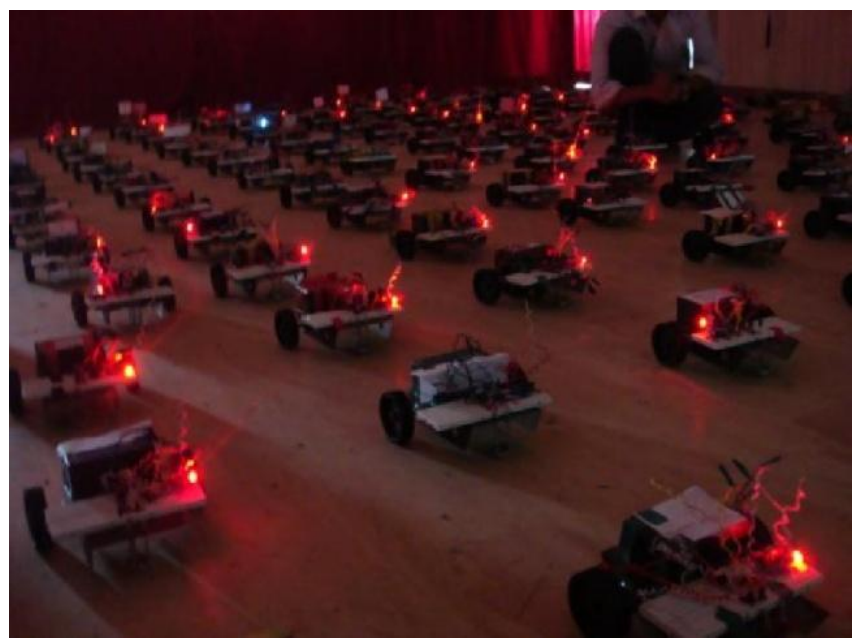

Fig 4: COMBOTS interacting with tutor

\section{MASTER/TUTOR COMBOT}

Apart from teaching the tasks to the student COMBOTs, it is also required by the tutor COMBOT to know the status of the COMBOT physiologically as well as psychologically. In case of robots, the physiological status determines its separation, cohesion and alignment with respect to its neighboring robots [9].

So to provide this information to the tutor, gray code concept can be used that would determine all the three parameters. This concept has not been discussed here in detail. Hence the student COMBOT transmits this information to the tutor at regular intervals.

In case of the psychological state, the student keeps a track of the no. of tasks learned, and makes the following calculation.

Capability ratio $=$ no. tasks learned/ total memory $\left(\sum \mathrm{m}\right)$

Where $\mathrm{m}=$ bits available in the memory of controlling unit.This code is transmitted which gives the idea of the memory status of the student

COMBOT. This code can also transmitted on demand by the tutor. This helps the tutor to realize the capability of an individual student COMBOT and the areas of its deficit. 


\section{APPLICATION}

The above research finds a huge scope in the education industry where each individual can be taught to represent himself as a student and build a robot. Robotics being an interdisciplinary subject, teaches a student all the fundamental concepts of engineering of the various branches. The student can not only have an in-depth knowledge of robot building but can also incorporate features that could represent him as an individual, that would also give him a better insight of himself, realizing his own capabilities.

Apart from the education industry it finds a huge application in space station and space colony building where a task maybe divided into several modules, each module being dealt by a cluster of robots. Further of its applications include automation industry, manufacturing plants, medical equipment handling.

\section{FUTURE WORK}

Although the above approach discussed here implements the concept of machine learning considerably, they still do not actually cause the COMBOT to be programmed wirelessly via the other COMBOT. This is the area where the future work is being directed and under progress. This concept would actually be implemented on one thousand (1000) robots to evolve this "classroom environment" into a "college environment" with different levels of hierarchy of machine or robot learning.

The work is going on for the development of a dynamically reconfigurable system which supports multiple modules running concurrently, all with hardware support. A standard Xilinx FPGA can be used to test the possibilities of loading partially new hardware configurations while other parts of the FPGA still are active. Dynamic Partial Reconfiguration (DPR) of FPGAs presents many opportunities for application design flexibility, enabling tasks to dynamically swap in and out of the FPGA without entire system interruption. Different modules can be prepared to perform different tasks defining these modules as partial reconfigurable module and main module as static module. The partial modules will be loaded dynamically toe the FPGA during run time. Using Partial dynamic reconfiguration feature of FPGA has many advantages like reduced power consumption. Reduced area as the size of the bitstream.which will be loaded in to memory will be less.

\section{REFERENCES}

[1]. Maze solving algorithms for Micromouse, swati mishra, pankaj bande, sitis 2008, IEEE ISBN: 978-0-7695-3493$0 / 08$.

[2] Jacques Ferber's, "Multi-Agent Systems: An Introduction to Distributed Artificial Intelligence" [Ferber 99]

[3] Arvin Agah, Chris T. Pierik "Design and fabrication of a team of robots in hardware", , AIST-MITI, ISBN 0-78032775-619 1996 IEEE
[4] Curt Bererton' and Pradeep K. Khosla "Towards A Team of Robots with Reconfiguration and Repair Capabilities “, proceedings of the 2001 IEEE International Conference on Robotics 8 Automation Seoul, Korea. May 21-26, 2001

[5] Pankaj bande, Navneet tohan and Swati mishra, "mobile controlled smart device for multiple device regulation", RAMPT'09, India

[6] Tom Mitchell, "Machine Learning", Mc-Graw Hill, ISBN: 007- 115467-1, 1997.

[7] Francisco J. Varela and Paul Bourgine, eds "Artificial Life and Real Robots "Rodney A. Brooks, "Toward a Practice of Autonomous Systems: Proceedings of the First European Conference on Artificial Life,"., MIT Press, Cambridge, MA, 1992,

[8] Maja J ,Mataric “A Distributed Model for Mobile Robot Environment-Learning and Navigation", MIT A.I. Lab Technical Report 1228, May.

[9] "What's different in learning in Multi Agent Systems?"Luís Nunes, Eugénio de Oliveira, Tech. Report ISCTE/NIAD\&R/FEUP, (apr/oct 2001)

[10] R. Pfeiffer, C. Scheier. Understanding Intelligence. MIT Press,Cambridge, MA. 1999.

[11] Behavioural Realism of Modelling Multi-Agent Communication Protocols in Virtual Environment ISBN 978-1-4244-3397-1/08/ O2008 IEEE Proc.7th Scandinavian Conference on Artificial Intelligence 2001

[12] Khaled Benkrid," High Performance Reconfigurable Computing:From Applications to Hardware" IAENG International Journal of Computer Science, 35:1, IJCS_35_1_04

[13] Yan Meng, Kerry Johnson, Brian Simms, and Matthew Conforth" A Modular-based Miniature Mobile Robot for Pervasive Computing" International Journal of Hybrid Information Technology Vol. 1, No. 1, January, 2008.

\section{AUTHORS PROFILE}

Mr. S.S.Shriramwar is working as a Assistant Professor at Priyadarshini College of Engineering. Nagpur, India. He has obtained his under graduate \& post graduate degree in Electronics Engineering from Nagpur University. Presently doing his $\mathrm{Ph} . \mathrm{D}$ in the area of dynamic reconfiguration of FPGA.

Dr. N.K.Choudhari is working as a principal at BCCE Nagpur, India. He has obtained his Ph.D. in Electronics Engineering. He is having more than 22 years of teaching experience with more than 20 publications in International journals and conferences. 